\title{
Biocompatible Gold Nanorod Conjugates for Preclinical Biomedical Research
}

\author{
Anton Liopo ${ }^{1 *}$, André Conjusteau ${ }^{1}$, Dmitri Tsyboulski ${ }^{1}$, Boris Ermolinsky², Alexander Kazansky² and Alexander Oraevsky ${ }^{1}$
}

${ }^{1}$ TomoWave Laboratories, Houston, TX 77081, USA

${ }^{2}$ Department of Biomedicine, University of Texas at Brownsville, TX 78520, USA

\begin{abstract}
Gold nanorods with a peak absorption wavelength of $760 \mathrm{~nm}$ were prepared using a seed-mediated method. A novel protocol has been developed to replace hexadecyltrimethylammonium bromide on the surface of the nanorods with 16-mercaptohexadecanoic acid and metoxy-poly(ethylene glycol)-thiol, and the monoclonal antibody HER2. The physical chemistry properties of the conjugates were monitored through optical and zeta-potential measurements to confirm surface chemistry changes. The efficiency of the modifications was quantified through measurement of the average number of antibodies per gold nanorod. The conjugates were investigated for different cells lines: BT-474, MCF7, MCF10, MDCK, and fibroblast. The results show successful cell accumulation of the gold nanorod HER2 conjugates in cells with HER2 overexpression. Incubation of the complexes in heparinized mouse blood demonstrated the low aggregation of the metallic particles through stability of the spectral properties, as verified by UV/VIS spectrometry. Cytotoxicity analysis with LDH release and MTT assay confirms strong targeting and retention of functional activity of the antibody after their conjugation with gold nanorods. Silver staining confirms efficient specific binding to BT-474 cells even in cases where the nanorod complexes were incubated in heparinized mouse blood. This is confirmed through in vivo studies where, following intravenous injection of gold nanorod complexes, silver staining reveals noticeably higher rates of specific binding in mouse tumors than in healthy liver.
\end{abstract}

The conjugates are reproducible, have strong molecular targeting capabilities, have long term stability in vivo and can be used in pre-clinical applications. The conjugates can also be used for molecular and optoacoustic imaging, quantitative sensing of biological substrates, and photothermal therapy.

Keywords: Gold nanorod fabrication; Metallic nanoparticles; Biocompatible targeting agent; Gold surface modification; Antibody conjugation; Cell particle targeting

\section{Introduction}

Gold nanoparticles of various shapes have promising applications for the biomedical field [1-5]. Gold nanoparticles (NPs) have become a focus of interest in the fields of nanobiotechnology and biomedicine because of the ease of surface bioconjugation with molecular probes as well as remarkable optical properties related to the Localized Surface Plasmon Resonance (LSPR) [2,6-8]. LSPR of gold nanoparticles results in strong optical absorption and scattering at visible and nearinfrared wavelengths $[6,9]$. One type of gold nanoparticle with a strong tunable plasmon resonance in the near-infrared spectral range is the gold nanorod (GNR) [6]. GNR can absorb light about one thousand times more strongly than an equivalent volume of an organic dye $[6,10]$. Consequently, GNR have been employed in diagnostics [11], therapeutic-delivery systems [12], including small-molecule drugs [13] and exogeneous nucleic acids [14], imaging [15,16], sensing [17], and responsive advanced material assemblies [18]. Properties such as biocompatibility ease of functionalization, and near infrared optical imaging make gold nanorods promising in new theranostic platforms [4]. GNRs were also used as optoacoustic (OA) contrast agents for quantitative flow analysis in biological tissues [19] and to investigate the kinetics of drug delivery compounds [20] or contrasting imaging [16]. GNR stabilized with cetyltrimethylammonium bromide (CTAB) shows cytotoxicity [21]. To reduce CTAB cytotoxicity, gold nanoparticles in the solution are washed by centrifugation [22]. However, GNR require pegylation for in vivo, and many other types of applications where the CTAB layer is being replaced by poly(ethylene glycol). [21,22]. The modification of GNR is usually performed by adding poly(ethylene glycol) thiol (r-PEG-SH, or simply PEG) to the CTAB coated GNR solution, followed by the removal of the excess
CTAB via dialysis or centrifugation $[4,20,21,23,24]$. The reasons for PEGylation of nanoparticle surface, i.e. the covalent attachment through thiol terminated PEG [25], includes shielding of antigenic and immunogenic epitopes, shielding receptor-mediated uptake by the Reticuloendothelial System (RES), and preventing recognition and degradation by proteolytic enzymes [26]. PEG-modified GNR showed a negative surface potential [22], as well as low level cytotoxicity in vitro [21], and can therefore be used for biomedical applications [27].

After intravenous administration, GNRs get distributed inside the body according to their modified affinity resulting in the enhancement of the targeted tissues [28]. The use of structurally modified GNR is less toxic to normal tissue during delivery. At the molecular level, GNR could traverse biologic barriers and preferentially accumulate in cancer cells [9, 29-31]. Targeting GNR to a specific site is a critical aspect of bioimaging when used as a contrast agent. It is also critical for achieving efficient photothermal therapy without side effects, especially after intravenous injection [32].

Methods for enhancing the accumulation in cells and tissues with strongly absorbing gold nanoparticles and gold nanorods were previously discussed $[2,4,5]$. The standard for conjugating antibodies

${ }^{*}$ Corresponding author: Anton Liopo, TomoWave Laboratories, 6550 Mapleridge St., Suite 124, Houston TX 77081-4629, USA, Tel: 1713270 5393; Fax: 1713270 5392; E-mail: al@tomowave.com

Received July 19, 2012; Accepted August 03, 2012; Published August 03, 2012

Citation: Liopo A, Conjusteau A, Tsyboulski D, Ermolinsky B, Kazansky A, et al. (2012) Biocompatible Gold Nanorod Conjugates for Preclinical Biomedical Research. J Nanomedic Nanotechnol 6:274. doi:10.4172/2157-7439.1000274

Copyright: ( 2012 Liopo A, et al. This is an open-access article distributed under the terms of the Creative Commons Attribution License, which permits unrestricted use, distribution, and reproduction in any medium, provided the original author and source are credited. 
to gold nanoparticles using covalent bonding was published by several research groups [1,4,33-35].

However, the conjugation processes are in need of improvement. Most protocols are hard to adapt to large-scale manufacturing of highly concentrated conjugates with strong affinity toward factors such as biochemical and physiological conditions of the cells and organs of the body [35].

In these studies, we adopted the published methodology of GNR fabrication $[34,36,37]$ to get a high yield of narrow band GNR with optical absorption centered at $760 \mathrm{~nm}$. The manufactured nanorods were pegylated and conjugated with monoclonal antibody (mAb) to become non-toxic as biocompatible agent. We characterized the conjugation efficiency of the monoclonal antibody (mAb) HER2/neu by measuring and comparing antibody binding of the GNRs before and after pegylation. We devised a novel protocol through reordering the steps involved in PEGylating GNR $\mathrm{mAB}$ conjugates for use in preclinical research with specific accumulation in tumors.

\section{Materials and Methods}

\section{Reagents}

The chemicals used in this study were purchased from the following companies: Hexadecyltrimethylammonium bromide (CTAB, Sigma), Gold(III) chloride trihydrate $\left(\mathrm{HAuCl}_{4} \cdot 3 \mathrm{H}_{2} \mathrm{O}\right.$, Aldrich), Sodium Borohydride $\left(\mathrm{NaBH}_{4}\right.$, Aldrich), Silver Nitrate $\left(\mathrm{AgNO}_{3}\right.$, Sigma-Aldrich), Ascorbic Acid (Sigma), Potassium carbonate $\left(\mathrm{K}_{2} \mathrm{CO}_{3}\right.$, Sigma- Aldrich), Poly (ethylene glycol) methyl ether thiol or Methoxypolyethylene glycol thiol mPEG thiol, MW 5000, (mPEG-Thiol or PEG, Laysan Bio Inc.), 16-Mercaptohexadecanoic acid (MHDA, Sigma), Sodium Chloride (NaCl, Sigma), 1-Ethyl-3-[3-dimethylaminopropyl] carbodiimide hydrochloride (EDC, Pierce), N-hydroxysulfosuccinimide (sulfoNHS, Pierce), 2-(N-Morpholino) ethane sulfonic acid sodium salt buffer (MES, Sigma-Aldrich), Monoclonal antibody Herceptin (HER2, Genentech), trypan blue (Sigma), Phosphate buffered saline (PBS, Sigma), tris-buffered saline with Tween-20 (TBST, Dako, Denmark), target retrieval solution (TRS, Dako, Denmark).

\section{Fabrication, PEGylation, conjugation of GNR, and characterization}

We present below the details of a GNR fabrication protocol adapted from previously reported methodology $[34,37,38]$. The base procedure is tailored to the needs of the specific experiments presented in this paper. It allows high-yield fabrication of a narrow size distribution of rods with a $760 \mathrm{~nm}$ plasmon resonance. In a typical procedure, 0.250 $\mathrm{ml}$ of an aqueous $0.01 \mathrm{M}$ solution of $\mathrm{HAuCl}_{4} \cdot 3 \mathrm{H}_{2} \mathrm{O}$ was added to $7.5 \mathrm{ml}$ of a $0.1 \mathrm{M} \mathrm{CTAB}$ solution in a test tube ( $15 \mathrm{ml}$ glass tube). Then, 0.600 $\mathrm{ml}$ of an aqueous $0.01 \mathrm{M}$ ice-cold $\mathrm{NaBH}_{4}$ solution was added all at once. This seed solution was used 2-3 hours after its preparation. In the next step of the fabrication, exact proportions of $9.5 \mathrm{~mL}$ of $0.10 \mathrm{M} \mathrm{CTAB}$, $0.400 \mathrm{ml}$ of $0.01 \mathrm{M} \mathrm{HAuCl}_{4} \cdot 3 \mathrm{H}_{2} \mathrm{O}$, and $0.060 \mathrm{ml}$ of $0.01 \mathrm{M} \mathrm{AgNO}_{3}$ solutions were added one at a time in the preceding order, then gently mixed by inversion. The solution at this stage appeared bright brownyellow in color. Then $0.064 \mathrm{ml}$ of $0.10 \mathrm{M}$ ascorbic acid was added. The solution became colorless upon addition and mixing of ascorbic acid. Ten minutes were allowed for the reaction to fully proceed before adding the required quantity of seed solution. The reaction mixture was gently mixed for 10 seconds and left undisturbed for 1-2 hours. Then, the solution was left under thermostatic conditions for 24 hours at the temperature of $30^{\circ} \mathrm{C}[1,37]$.
Before covalent binding with PEG, or conjugation with monoclonal antibody HER2, the GNR were centrifuged at low speed $(1500 \times \mathrm{g}, 20$ $\min$ ) for separation of unwanted aggregates like platelets and stars. Figure 1 shows UV-VIS absorption spectra of GNR. The first fraction comes from resuspension of the pellet after low speed centrifugation of the GNR-CTAB stock solution (not used in this report). The second fraction is from the supernatant which was used in all experiments. Spectra are normalized to match the short wavelength peak of the GNR-CTAB supernatant data. The GNR-CTAB complex is now ready for further modification. For pegylation [21,34], the GNR-CTAB solution was centrifuged at $14000 \mathrm{~g}$ for 10 minutes, the supernatant was removed, and the pellet was resuspended in Milli Q Water (MQW) to reduce the residual CTAB concentration to $0.01 \mathrm{M}$. Then, $1.0 \mathrm{ml}$ of $2 \mathrm{mM} \mathrm{K}_{2} \mathrm{CO}_{3}$ was added to $8 \mathrm{ml}$ of aqueous GNR solution and 1.0 $\mathrm{ml}$ of $0.1 \mathrm{mM}$ mPEG-Thiol (PEG). The resulting mixture was kept on a rocking platform at room temperature overnight. Excess PEG was removed from the solution by two rounds of centrifugation prior to final resuspension in PBS ( $\mathrm{pH}$ 7.4).

For conjugation with monoclonal antibody, the general procedure replaces $\mathrm{CTAB}$ as described in the literature $[21,33]$ on the surface of GNR with MHDA for activation [34,38], monoclonal antibody (HER2, purified using desalting columns (Pierce Biotechnology, Rockford, IL) or a Microcon centrifugal filter device from Millipore), and PEG [21]. Figure 2 presents a schematic of the conjugation protocols investigated in this work. They differ by the order of the surface modification steps.

\section{Protocol 1}

The GNR-CTAB complex was resuspended in $1 \mathrm{ml}$ of MQW. The GNR solution was then added to solutions of MHDA and PEG in molar ratio of 1 to 4 (or $2 \mathrm{mM}$ and $8 \mathrm{mM}$ respectively). This molar ratio was found experimentally: the optimal ratio was chosen as maximizing the area covered by the linker MHDA, therefore increasing the likelihood

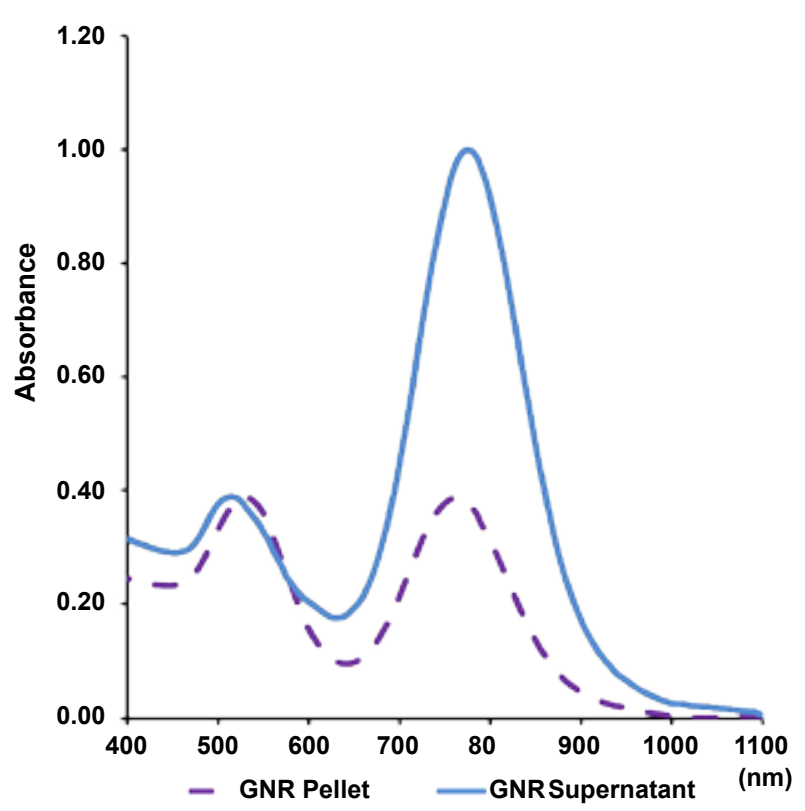

Figure 1: UV-VIS absorption spectra of GNR. First fraction is from resuspension of the pellet after low speed centrifugation of GNR-CTAB stock solution (not used in this report). The second fraction is from the supernatant which was use in all experiments. Spectra are normalized to match the short wavelength peak of the GNR-CTAB supernatant data. 


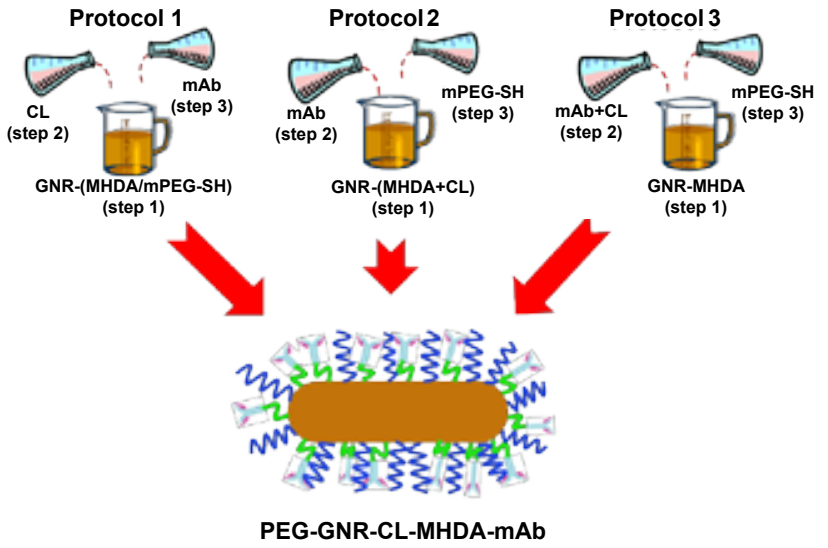

Figure 2: Different protocols for conjugation of GNR with antibody.

of binding with the antibody, while maintaining the plasmon resonance of the control (GNR-PEG). The spectra are presented in Figure 3: UV-VIS absorption spectra of GNR-Ab (HER2) conjugates with different modifications of GNR surface after incubation at 3 hours in $10 \%$ solution of FBS. Control sample is pegylated GNR (PEG). The other three samples are GNR modified with MHDA and PEG (molar ratios are 1, 2 and 4 MHDA for 8 PEG: $1 \mathrm{M}: 8 \mathrm{P}, 2 \mathrm{M}: 8 \mathrm{P}$ and $4 \mathrm{M}: 8 \mathrm{P}$, respectively), activated with cross linkers EDC and sulfo NHS (CL), then conjugated with Ab HER2.

The heterofunctional linker MHDA was added drop wise to GNR stock solution under mixing $(2.5 \mu \mathrm{l}$ of $2 \mathrm{mM}$ MHDA for each $1 \mathrm{ml}$ solution of GNR-CTAB, concentration $500 \mathrm{pM}$ or ratio is 1:10000). After 60 min of mixing at RT, the GNR were exposed to a solution mPEG Thiol $(2.5 \mu \mathrm{l}$ of $8 \mathrm{mM}$ PEG for each $1 \mathrm{ml}$ solution of GNRMHDA: ratio is 1:40000) overnight as presented above and reported in the literature $[21,34]$. In our experience, this step can be significantly shortened with no noticeable ill effects. 4-6 hours at RT was found to be enough for efficient removal of CTAB.

This last step ensures filling the areas not covered by the heterofunctional MHDA, yielding well-shielded and stable particle surfaces. Before activation with EDC and sulfo NHS the GNR were purified by two rounds of centrifugation $(12,000 \times \mathrm{g}, 15 \mathrm{~min}$ each), the supernatant was removed, and the pellet was resuspended in $1 \mathrm{ml}$ of MQW. $10 \mu \mathrm{l}$ of a mixture of EDC and sulfo-NHS were added from stock solution in MES buffer (0.1 M, pH 6.0) with $0.5 \mathrm{M} \mathrm{NaCl}$ in $10 \mathrm{mM}$ and $0.4 \mathrm{mM}$ concentrations, respectively. The resulting solution was gently agitated for $30 \mathrm{~min}$ at RT. The solution was again centrifuged at $12000 \mathrm{RPM}$ for 10 minutes, the supernatant was removed and $\mathrm{mAb}$ HER2 was added to a final concentration of $100 \mu \mathrm{g} / \mathrm{ml}$ to $1 \mathrm{ml}$ of 500 $\mathrm{pM}$ activated GNR. This represents a ratio around 1000 between the number of $\mathrm{Ab}$ molecules and GNR. The mixture was incubated at RT for 1 hour and then left on a rocking platform overnight $\left(+4^{\circ} \mathrm{C}\right)$. The final step was centrifugation of the solution of conjugates PEG-GNR$\mathrm{mAb}$ at $12000 \mathrm{~g}$ for 10 minutes. After resuspension, the pegylation step described above was repeated for $2 \mathrm{~h}$ at RT.

Then, the supernatant was removed and the pellet was resuspended in PBS ( $\mathrm{pH}$ 7.4) and the conjugates were filtered through a $0.45 \mu \mathrm{m}$ Millipore Express Plus membrane. The required concentration, determined from the molar extinction of our GNR $\left(3.85 \times 10^{9}\right.$ $\mathrm{M}^{-1} \mathrm{~cm}^{-1}$ ), was confirmed by optical density measurement and the spectral properties (UV-VIS spectra into range 400-1100 nm) were monitored by Beckman 530 or Thermo Scientific Evolution 201 spectrophotometer[39].

\section{Protocol 2}

The second protocol (Protocol 2, Figure 2) was described before $[33,34]$ but this work reports have slight modifications. For this, one $\mathrm{ml}$ of synthesized GNR in CTAB was centrifuged twice in a 1.5 $\mathrm{mL}$ eppendorf tube at $14000 \mathrm{RPM}$ for 15 minutes and resuspended in one $\mathrm{mL}$ of MQW to a concentration of $500 \mathrm{pM}$. Then, $10 \mu \mathrm{L}$ of 5 $\mathrm{mM}$ MHDA in ethanol was added to the GNR and the solution was sonicated for 30 minutes at $50^{\circ} \mathrm{C}$ to prevent aggregation. The solution was centrifuged at 12000 RPM for 10 minutes, the supernatant was removed, and the pellet was resuspended in MQW. $10 \mu \mathrm{LDC}$ and sulfo-NHS was added from stock solution in MES buffer in $10 \mathrm{mM}$ and $0.4 \mathrm{mM}$ concentrations, respectively. The mixture was sonicated for 30 minutes at room temperature (RT) to produce activated GNR (GNR that are capable of binding to the amine side chain of proteins). HER2 was added, at a final concentration of $100 \mu \mathrm{g} / \mathrm{ml}$, to $1 \mathrm{ml}$ of 500 pM activated GNR just like in Protocol 1. The mixture was sonicated at RT for 30min and then left on a rocking platform overnight. Following the removal of excess HER2, $10 \mu \mathrm{l}$ of PEG $(1 \mathrm{mM})$ was added to $1 \mathrm{ml}$ of GNR-HER2 conjugates and the mixture was incubated at room

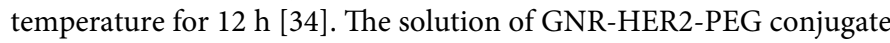
was centrifuged at $12000 \mathrm{~g}$ for 10 minutes, the supernatant was removed and the pellet was resuspended in PBS pH 7.4 to a working concentration determined spectrophotometrically.

\section{Protocol 3}

The third method of activation (Protocol 3) has the modification steps performed in another order (Figure2). Same one $\mathrm{mL}$ of synthesized GNR in CTAB was resuspended in one $\mathrm{mL}$ of MQW. MHDA was added to the GNR-CTAB solution, and the solution was sonicated for 30 minutes at $50^{\circ} \mathrm{C}$ to prevent aggregation. The solution

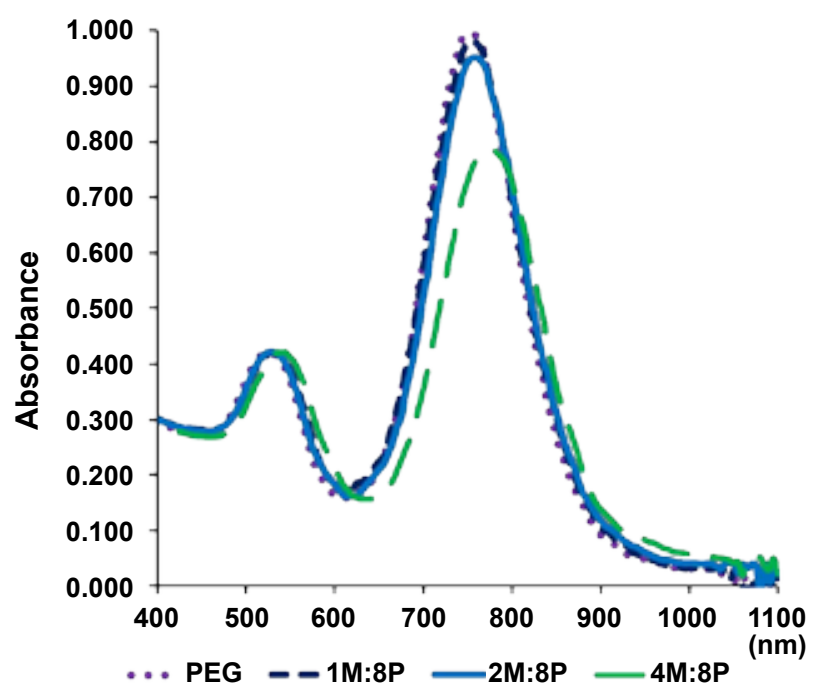

Figure 3: UV-VIS absorption spectra of GNR-Ab (HER2) conjugates with different modifications of GNR surface after incubation at 3 hours in $10 \%$ solution of FBS. Control sample is pegylated GNR (PEG). The other three samples are GNR modified with MHDA and PEG (molar ratios are 1,2 and 4 MHDA for 8 PEG: $1 \mathrm{M}: 8 \mathrm{P}, 2 \mathrm{M}: 8 \mathrm{P}$ and $4 \mathrm{M}: 8 \mathrm{P}$, respectively), activated with cross linkers EDC and sulfo NHS (CL), then conjugated with Ab HER2. 
was centrifuged at 12000 RPM for 10 minutes, the supernatant was removed and the pellet was resuspended in MQW. At the same time a complex of $\mathrm{mAb}$ and crosslinkers was prepared from $\mathrm{mAb}$ and solution of EDC/sulfo NHS in MES buffer. The compound was agitated at RT for $30 \mathrm{~min}$. The activated $\mathrm{mAb}$ was purified by centrifugation with 3000 $\mathrm{kDa}$ membrane (possible use dialysis), and additional washing by salt column (Pierce). The clean complex was added to the GNR-MHDA solution and left overnight $\left(4^{\circ} \mathrm{C}\right)$. Following the removal of excess $\mathrm{mAb}$ by centrifugation, the GNR-mAb complex was pegylated as shown in Protocol 2. The resulting solution of GNR-mAb-PEG conjugate was centrifuged at $12000 \mathrm{~g}$ for 10 minutes, the supernatant was removed and the pellet was resuspended in PBS pH 7.4 to a working concentration determined spectrophotometrically.

The zeta-potential of GNR before and after formation of different conjugates was measured with a high performance particle sizer (Malvern Instruments Ltd., Southborough, MA, USA) at $25^{\circ} \mathrm{C}$, and ten 20 -second runs were performed for each sample $[39,40]$. Zeta-potential is a measure of both particle stability and adhesion. More negative or positive values of zeta-potential are associated with more stable particle solution, because repulsion between the particles reduces the particle aggregation $[38,40]$. (Figure 4) Zeta-Potential $(\mathrm{mV})$ for CTAB coated GNR stock solution (CTAB), GNR after PEGylation (PEG), GNR after removed CTAB with MHDA and PEG (MHDA-PEG) and three different protocols for conjugation and pegylation. GNR with MHDA and PEG, activated with cross linkers EDC and sulfo NHS (CL) and conjugated with Ab: GNR-MHDA/PEG+CL+A (Prot 1); GNR with MHDA, activated with $\mathrm{CL}$, conjugated with $\mathrm{Ab}$ and pegylated: GNRMHDA+CL+Ab+PEG (Prot 2); GNR with MHDA, conjugated with Ab CL complex and pegylated: GNR+MHDA+CL/Ab+PEG (Prot 3) (mean \pm SEM, $\mathrm{n}=10$-14 for each conjugate).

A measure of total and bound protein (HER2) was performed with the Pierce Micro BCA ${ }^{\mathrm{Ts}}$ Protein Assay Reagent Kit (Pierce). Concentration of HER2 was measured before, and after addition of GNR-activated solution: it is dependent upon either the level of monoclonal antibody, or incubation time. Figure 5 shows Mean number of HER2 molecules on the surface of GNR after conjugation through different protocols (synthesis steps for protocol 1,2 and 3 are the same as in Figure 4). As negative control we used solutions of antibody without GNR. The determination was performed through measurement of absorbance at or near $562 \mathrm{~nm}$ by Cary 500 UV-VISNIR spectrophotometer [38]. It is important to note that the ratio of absorbance at $562 \mathrm{~nm}$ (proteins relative to BSA) has a coefficient of variation of only around 10\% [39].

\section{Cell culture, viability and cell proliferation}

Cell lines BT 474 (Human breast distal carcinoma with HER2 receptor overexpression), MCF 7 (Human breast adenocarcinoma with HER2 receptor overexpression), MCF 10A (Human breast fibrocystic disease, no HER2 receptor overexpression), MDCK (normal dog kidney epithelial cells), and Human lung normal fibroblast were obtained from American Type Culture Collection (ATCC) and cultured in essential media with $10 \%$ fetal bovine serum.

Cell viability (Figure 6) was determined using the Cytotoxicity Detection Kit which measures cytotoxicity by detecting lactate dehydrogenase (LDH) activity released from damaged cells (Roche). It was described in our previous works $[40,41]$.

Cell survival following GNR conjugates incubation at different concentrations (50-500 pM) was monitored. BT 474 cells were seeded into a 96-well plate at a density of $2 \times 10^{5}$ cells $/ \mathrm{ml}$ in $0.1 \mathrm{ml}$ of media per well. 3 hours before treatment, media were changed to $0.1 \mathrm{ml}$ of low serum (0.5\%). $0.025 \mathrm{ml}$ of GNR conjugates were added in low serum media too. After $48 \mathrm{~h}$ of incubation with GNR conjugates, the $25 \mu \mathrm{l}$ samples of media were collected for measurement of $\mathrm{LDH}$ release from the cells $[40,41]$.

The proliferation of viable cells was determined by examining the conversion of MTT [3-(4,5-Dimethylthiazol-2-yl)-2,5-diphenyltetrazolium bromide, a tetrazole] to a purple formazan product by metabolically active cells using a kit (Roche). BT 474 cells were used in the same plate (after removing the $25 \mu \mathrm{l}$ of media for LDH assay) and the MTT assay was performed on the pretreated plate with different GNR cells. Absorbance of the LDH and MTT products was measured on a plate spectrophotometer (Bio-Tek Instruments, Inc.). Data for LDH release, MTT assay and LDH/MTT ratios, were measured and calculated from 4-6 replicates. Figure 7 summarizes the results: Metabolically active cells (MTT assay), LDH release, and ratio between $\mathrm{LDH}$ release

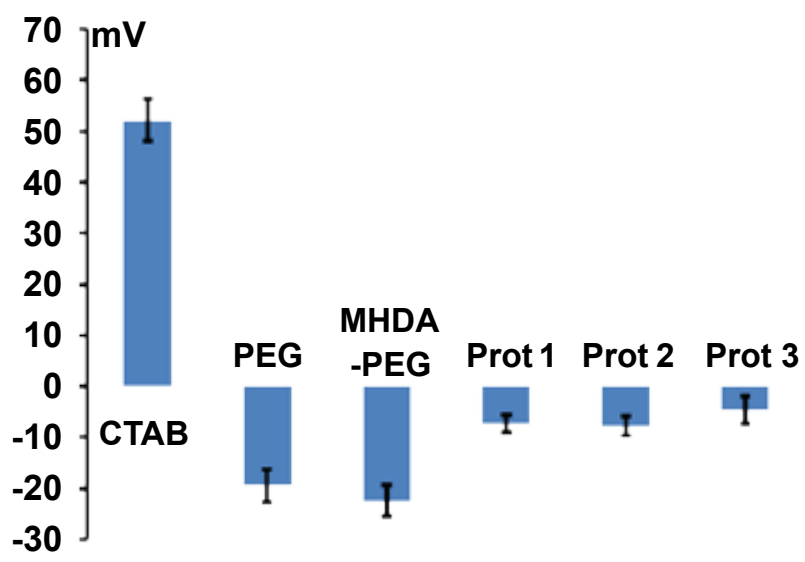

Figure 4: Zeta-Potential ( $\mathrm{mV}$ ) for CTAB coated GNR stock solution (CTAB) GNR after PEGylation (PEG), GNR after removed CTAB with MHDA and PEG (MHDA-PEG) and three different protocols for conjugation and pegylation. GNR with MHDA and PEG, activated with cross linkers EDC and sulfo NHS (CL) and conjugated with Ab: GNR-MHDA/PEG+CL+A (Prot 1); GNR with MHDA, activated with $C L$, conjugated with $A b$ and pegylated: GNR$M H D A+C L+A b+P E G$ (Prot 2); GNR with MHDA, conjugated with Ab CL complex and pegylated: GNR+MHDA+CL/Ab+PEG (Prot 3 ) (mean \pm SEM, $n=10-14$ for each conjugate)

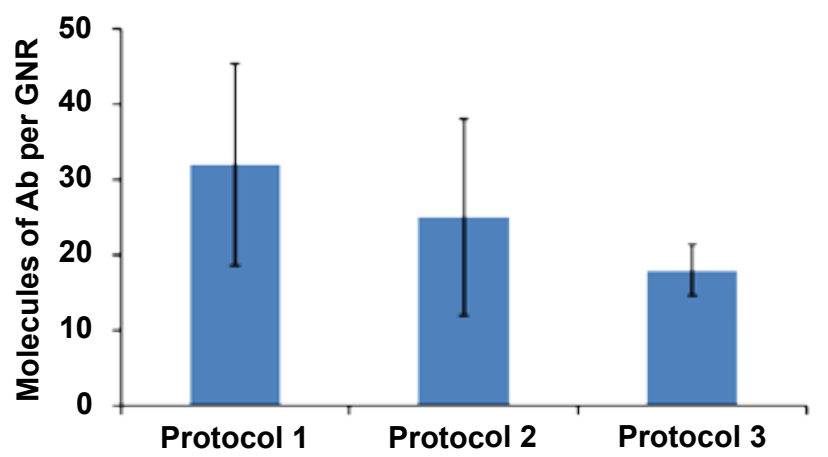

Figure 5: Mean number of HER2 molecules on the surface of GNR after conjugation through different protocols (synthesis steps for protocol 1, 2 and 3 are the same as in Figure 4). 


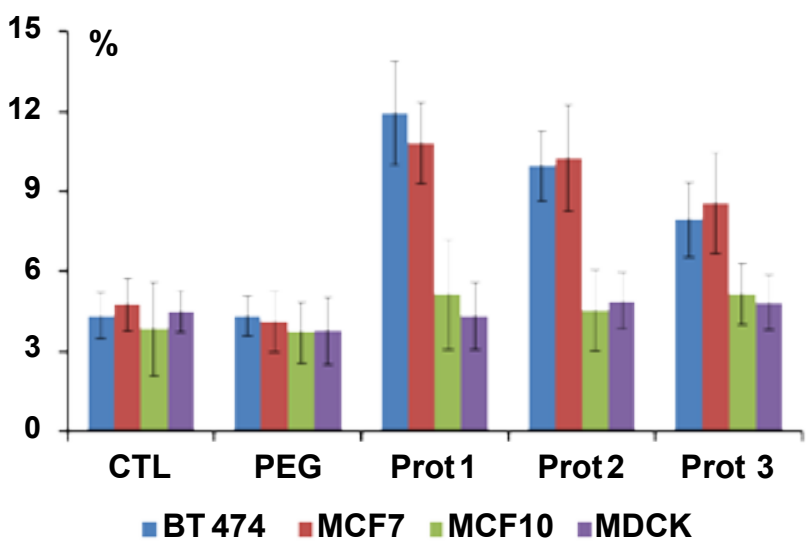

Figure 6: Fraction of dead cells for the cell lines (BT 474, MCF 7, MCF 10 and MDCK) after incubation with GNR HER2 conjugates for $48 \mathrm{~h}, 250 \mathrm{pM}$ (or $1.5 \times 10^{11} \mathrm{GNR} / \mathrm{ml}$ ). The number of dead cells was counted after staining with Trypan Blue. Control groups of cells received treatment with PBS or GNR after PEGylation (PEG). Synthesis steps for protocols 1, 2 and 3 are the same as in Figure 4 (mean $\pm S D, n=6$ independent measures for each conjugate). Results indicate significantly higher level of cell death for cells with HER2/neu expression, BT 474 and MCF7, and no significant changes without HER2/neu expression: MCF 10 and MDCK.

and MTT following incubation of BT 474 cell with GNR conjugates for $48 \mathrm{~h}$, at concentrations up to $500 \mathrm{pM}$ (or $3 \times 10^{11} \mathrm{GNR} / \mathrm{ml}$ ). Synthesis steps for protocols 1, 2 and 3 are the same as in Figure 4 (mean \pm SD, $\mathrm{n}=6$ independent measures for each conjugate). All protocols show significant level of cell death, from Prot 1 (highest) to Prot 3 (lowest).

The other technique to assess cell viability is based on trypan blue (Sigma) dye exclusion [40]. This staining was used for investigation of cell viability of BT 474, MCF 7, MCF 10 and MDCK cells as well as for measuring surviving these cells their after incubation with GNRPEG, and conjugates from protocols 1,2 and 3 (all at $500 \mathrm{pM}$ or $3 \times$ $\left.10^{11} \mathrm{GNR} / \mathrm{ml}, 48 \mathrm{~h}, \mathrm{n}=6, \mathrm{M} \pm \mathrm{SD}\right)$. After this, the cells were incubated 5 min with $0.4 \%$ trypan blue, and counted as a percentage of stained cells to total number of cells. As positive control, we used cells to which only PBS solution ( $\mathrm{pH} 7.4$, at $25^{\circ} \mathrm{C}$ ) was added. Correspondingly, knowingly toxic CTAB-coated GNR solutions [16] were added in the same concentrations as GNR conjugates and these samples were used as negative controls in each experimental condition.

This thorough comparative study had been devised in order to compare the GNR conjugates fabricated with 3 different protocols, and assess their relative performance. After confirming suitability, the novel order of conjugation (Protocol 1) was chosen for all in vitro and in vivo experiments related to the visualization of receptors and distribution on GNR conjugates in tumor and liver tissue.

Optical visualization of binding of GNR conjugates with BT 474 cells and fibroblasts was performed through the use of a silver staining kit (SS, BBI International, UK) according to manufacturer instructions.

Cells with (BT 474) and without (Fibroblasts) overexpression of HER2 receptors were treated with GNR HER2 conjugates. Incubation time of GNR conjugates was $1 \mathrm{~h}$. GNR conjugates were pretreated with heparinized mouse blood (preincubation time is $4 \mathrm{~h}$ ). Optical visualization of HER2 receptors was performed after fixation of cells with a mixture of formaldehyde (2.5\%) and glutaraldehyde (1.5\%). Figure 9 shows Silver staining of fibroblast (no HER2/neu expression) and BT-474 cells following 60 min pre-treatment with pegylated (GNRPEG) or conjugated through protocol 1 (GNR-PEG-HER2) GNR which were incubated with heparinized mouse blood for four hours. BT 474 shows silver enhancement for GNR-PEG-HER2 conjugates in both conditions: before and after incubation of GNR-conjugates with blood.

\section{Animal studies for optical and optoacoustic imaging}

For investigation of GNR HER2 conjugates distribution we used (animal models) mice with tumors that overexpressed HER2/neu receptor. In vivo experiments aimed to evaluate whether GNR HER2 conjugates are accumulated within tumors that overexpress HER2/ neu receptor in the same as in other animal model tissue without overexpression of this receptor. We used Athymic Nude-Foxn $1^{\text {nu }}$ mice (Harlan), 7-9 weeks old, weighing about $25 \mathrm{~g}$. Animal handling, GNR conjugates injections, and euthanasia were described in detail in our publications $[15,16]$ and each mouse-related procedure was in compliance with our Institutional Animal Care and Use Committee (IACUC) protocol. This model was made through BT474 cells injection $\left(2 \times 10^{6}\right)$, subcutaneously in the flank area of nude mice [34]. The tumors had a diameter of 4-6 $\mathrm{mm}$ after three-four weeks.

The mice had $200 \mu \mathrm{l}$ of GNR conjugates in sterile PBS injected intravenously (IV) through the tail vein. The injected solution contained $7.5 \times 10^{12} \mathrm{GNR} / \mathrm{ml}$ or $12.5 \mathrm{nM}$, which is equivalent to 100 -

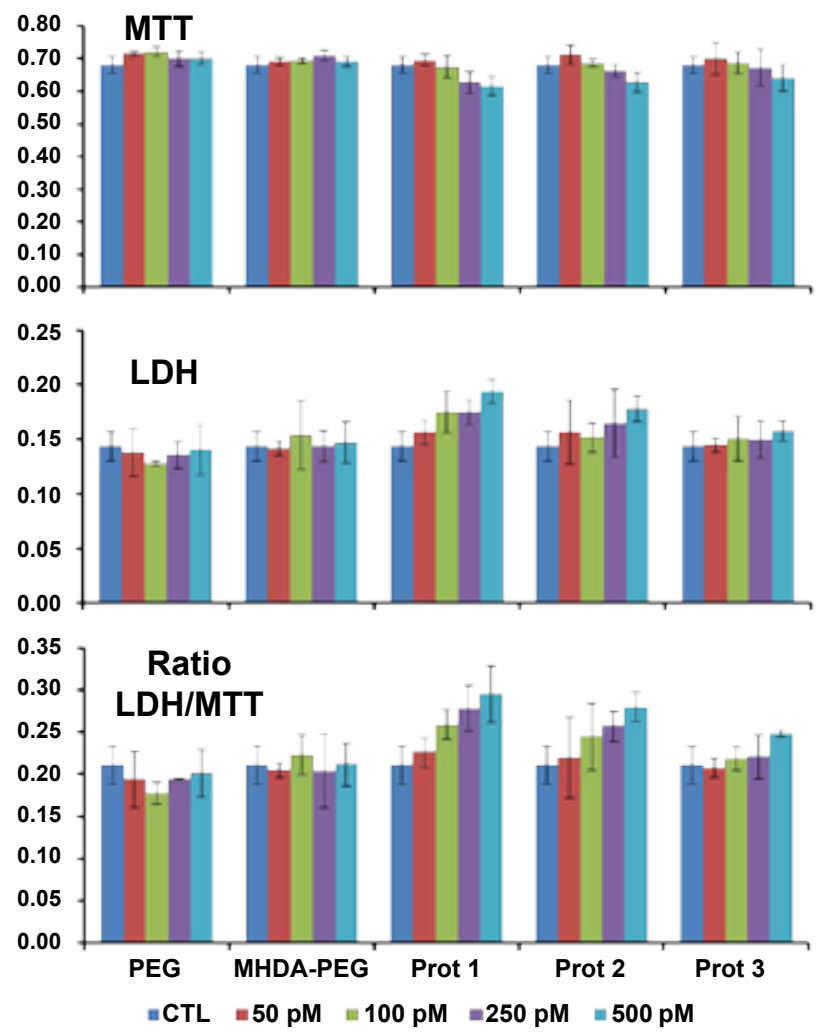

Figure 7: Metabolically active cells (MTT assay), LDH release, and ratio between LDH release and MTT following incubation of BT 474 cell with GNR conjugates for $48 \mathrm{~h}$, at concentrations up to $500 \mathrm{pM}$ (or $3 \times 10^{11} \mathrm{GNR} / \mathrm{ml}$ ). Synthesis steps for protocols 1,2 and 3 are the same as in figure 4 (mean $\pm \mathrm{SD}, \mathrm{n}=6$ independent measures for each conjugate). All protocols show significant level of cell death, from Prot 1 (highest) to Prot 3 (lowest). 
$150 \mathrm{pM}$ after distribution within the mouse's body. Selected mice were sacrificed at $48 \mathrm{~h}$ after the IV injections of GNR conjugates. Tissues of liver and tumor extractions were produced as paraffin-imbedded slices for Silver Staining (SS) and Hematoxylin Eosin (H\&E) staining. Tissue sections $(5 \mu \mathrm{m})$ were deparaffinized and rehydrated through xylene $(3$ changes, $5 \mathrm{~min}$ each) and graded ethanol solutions from 100 to $50 \%$ ( 1 min each). After this, samples were rinsed in $\mathrm{dH}_{2} \mathrm{O}$ and placed in a water bath for 10 min with tris-buffered saline with Tween 20 (TBST, Dako, Denmark). Retrieval with Target Retrieval Solution pH 6.1 (TRS, Dako, Denmark) was then performed in a preheated container at $96-99^{\circ} \mathrm{C}$ for $30-40 \mathrm{~min}$. The slides of liver sections were stained for PEG-GNR optical visualization with a SS Kit (BBI International, UK) according to manufacturer instructions, and HE stained for analysis of possible pathological consequences in liver after GNR conjugates administration. The results are shown in Figure 10 Above: Hematoxylin $\&$ Eosin (tumor and liver), and below: silver staining (tumor and liver) of GNR accumulated in mouse tissues following intravenous injection of PBS, GNR-PEG or GNR-PEG-HER2 conjugates. Silver staining shows that GNR peg or Ab conjugates have uniform distribution in liver tissue and noticeably higher numbers of GNR specific conjugates in mouse tumors.

\section{Result and Discussion}

We evaluated the protocol that improves the conjugation process of $\mathrm{mAb}$ to GNR and enhances GNR activity toward targeting antibodies. A significant part of this evaluation consists in the purification of GNR-CTAB stock solution. After low speed centrifugation of the stock solution, the pellet containing mostly platelets, stars, and other nonrod-like particles was discarded and only the supernatant was used as clean solution of GNR in different modification and conjugations procedures (Figure 1). In this study we compare three different protocols of activation and conjugation of GNR with $\mathrm{mAb}$ (Figure 2).

The optimized method of activation of GNR differs by the order of the modification steps (Protocol 1 on Figure 2). The GNR-CTAB MQW solution is mixed with MHDA and PEG (in molar ratio 1:4), and activated though EDC and sulfo-NHS as the complex PEG-GNRMHDA. This complex is capable of conjugation through the carboxy group of MHDA for binding with an amine function of proteins, antibody or peptides $[4,18]$. Optimization of the ratio between MHDA and PEG was investigated by UV-VIS absorption spectra of GNR-Ab (HER2) conjugates with different modifications of GNR surface after incubation at 3 hours in $10 \%$ solution of FBS (Figure 3). Conjugated HER2 on the surface modified with MHDA and PEG was more stable at 1 and 2 molar parts of MHDA for 8 parts of mPEG thiol. Due to the two fold increase in number of binding sites with similar stability in FBS solution, our choice was 1:4 molar ratio. This type of conjugation has not shown significantly different spectral behavior from pegylated GNR.

To confirm the chemistry modification of GNR-PEG conjugates we investigated the Zeta-potential (Figure 4). It was measured for CTAB coated GNR stock solution (CTAB), GNR after PEGylation (PEG), GNR after CTAB removal with MHDA and PEG (MHDA-PEG) and three different protocols for conjugation and pegylation. GNR with MHDA and PEG, activated with cross linkers EDC and sulfo NHS (CL) and conjugated with $\mathrm{Ab}$ : GNR-MHDA/PEG+CL+A (Prot 1); GNR with MHDA, activated with $\mathrm{CL}$, conjugated with $\mathrm{Ab}$ and pegylated: GNR-MHDA+CL+Ab+PEG (Prot 2); GNR with MHDA, conjugated with Ab CL complex and pegylated: GNR+MHDA+CL/Ab+PEG (Prot 3). The zeta-potential (Figure 4 ) of the GNR-CTAB complex was highly positive due to the presence of the positively charged CTAB molecules. After PEGylation, a negative zeta-potential confirmed chemistry changes to the GNR surface: the CTAB bi-layer was removed (Figure 4). The GNR HER 2 conjugates solution showed a zeta-potential which is slightly negative, but significantly different from zero. Zeta potential changes do confirm surface chemistry modifications, and correspond to previously published data $[40,42]$.

Investigation of binding of $\mathrm{mAb}$ (HER2) on the surface of activated GNR is presented in Figure 5 for all the protocols. Comparison of the different protocols of conjugation with number of HER2 molecules on the surface of GNR after conjugation shown that Protocol 1 yields the highest density of HER2 on the surface of GNR.

We investigated the influence of concentrations of GNR-PEG conjugates on the physiological status of cell cultures for different protocols of GNR conjugations (Figure 6). The cells of four lines (BT 474, MCF 7, MCF 10 and MDCK) were incubated with GNR HER2 conjugates for $48 \mathrm{~h}$, at $250 \mathrm{pM}$ (or $1.5 \times 10^{11} \mathrm{GNR} / \mathrm{ml}$ ). The number of dead cells was counted after staining with Trypan Blue [43]. Control groups of cells received treatment with PBS or GNR after PEGylation (PEG). Results indicate significantly higher level of cell death for cells with HER2/neu expression: BT 474 and MCF7. A trend toward increase

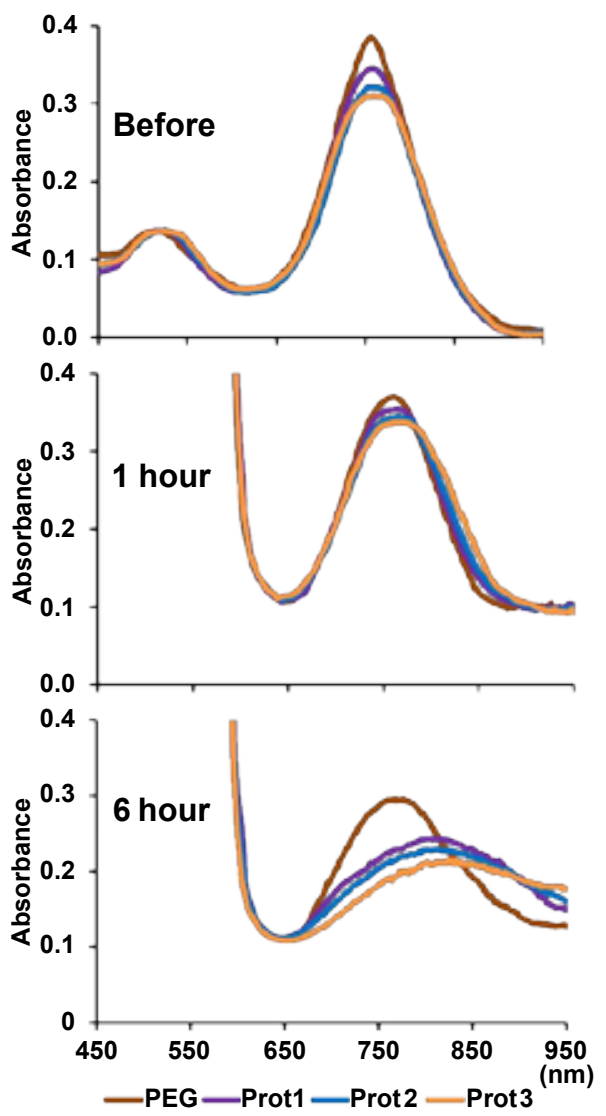

Figure 8: UV-VIS absorption spectra of supernatant solutions of GNR-Ab (HER2) conjugates after incubation for six hours in heparinized mouse blood. Synthesis steps for protocols 1,2 and 3 are the same as in figure 4 . Before and after $1 \mathrm{~h}$ incubation all protocols for conjugated and pegylated (PEG and Prot. 1, Prot. 2 and Prot. 3, respectively) have similar spectra. After $6 \mathrm{~h}$ incubations a higher level of aggregation is observed in samples from Prot 3 , with the best results for Prot.1 (lower aggregation) 
Citation: Liopo A, Conjusteau A, Tsyboulski D, Ermolinsky B, Kazansky A, et al. (2012) Biocompatible Gold Nanorod Conjugates for Preclinical Biomedical Research. J Nanomedic Nanotechnol 6:274. doi:10.4172/2157-7439.1000.274

of cell death can be seen for the cell lines without HER2/neu expression: MCF 10 and MDCK. Following GNR-CTAB administration, the level of dead cells was 4-5 times higher in comparison with GNR conjugates and did not show differentiation between groups of cells (data not presented).

Dose dependence response from BT 474 (overexpressed of HER2 receptors) was investigated by MTT assay, LDH release from the cell into the media, and ratio between LDH release and MTT following cell incubation with GNR conjugates for $48 \mathrm{~h}$, at concentrations up to 500 pM (Figure 7). All protocols show significant level of cell death, which corresponds to ratio of LDH by MTT $[16,44]$. However consequences for cell's, cell death was higher from Protocol 1 conjugates and lowest from Protocol 3.

Figure 8 shows UV-VIS absorption spectrum of supernatant solutions of GNR-Ab (HER2) conjugates after incubation for six hours in heparinized mouse blood. Before and after $1 \mathrm{~h}$ incubation. All protocols for conjugated and pegylated (PEG and Prot. 1, Prot. 2 and Prot. 3, respectively) have similar spectra. After $6 \mathrm{~h}$ incubation period, the pegylated sample shows little change, but a higher level of aggregation is observed in the conjugated samples, the highest from Protocol 3, and the lowest (best) results for conjugates from Protocol 1. This type of conjugate is more stable, resulting in an absorption peak around that of the control pegylated GNR, and can thus maintain the plasmon resonance in the biological transparency window for longer times $[6,21]$.

Comparative studies confirmed suitability of the novel order of conjugation steps, therefore Protocol 1 was used for experiments related to the characterization of specific targeting of GNR HER2 conjugates with cells in vivo application.

The incubation of GNR-PEG-HER2 conjugates with BT 474 and fibroblasts has shown to selectively bind to cancer cells after they are incubated in blood (Figure 9). GNR conjugates were incubated with heparinized mouse blood for $4 \mathrm{~h}$ and then were incubated with both BT- 474 cells as well as fibroblasts for $30 \mathrm{~min}$ at $37^{\circ} \mathrm{C}$. The cell plates were rinsed with PBS to remove unattached GNRs, and the plates were stained using silver enhancement kit. Figure 9 shows a significant increase in the number of GNR conjugates on the cells surface only for selective binding to BT-474 cells that overexpress HER2/neu receptor, but for both experimental conditions: before and after pre incubation of conjugates with blood. Fibroblasts do not have overexpressed HER2/ neu receptors and silver staining cannot visualize GNR conjugates on the cell surface. GNR conjugates were added to the media at a concentration of $500 \mathrm{pM}$. This concentration is consistent with reported levels of GNR-PEG in blood or tissue after IV administration in vivo $(12,22,23 \mathrm{ml})$. Results from these experiments are very similar to data previously published for methods of conjugation similar to Protocol 2 [34]. However, the novel method is much easier to perform, less time-consuming and provides a higher yield of conjugated GNR.

In vivo GNR conjugate administration has aimed to evaluate specific accumulation within tumors that overexpress HER2/neu receptors in animal models. Figure 10 shows results from an experiment in which GNR-PEG-HER2 complexes were found to accumulate within tumors and the liver of animals. 48 hours post tail vein injection, mice were sacrificed and samples of tumor and liver were stained with H\&E and SS (Figure 10). One group of mice was injected by GNR-PEG-HER2 conjugate, and another with pegylated GNR (GNR-PEG). One mouse was used as a control and thus was injected with only PBS. HE staining

(above) and SS (below) show different cases of GNR accumulated in mouse tissue following intravenous injection of PBS, GNR-PEG or GNR-PEG-HER2 conjugates. HE staining did not show a visible difference between the PBS control and the GNR slices of liver tissue after IV administration of GNR-PEG conjugates (Figure 10). The results are consistent with those reported by other groups [32,45] for similar dosage of GNR (around 10-20 mg/ $\mathrm{kg}$ body mass), and are presented solely as confirmation our GNR-PEG complexes are nontoxic. Silver staining shows that GNR PEG or Ab conjugates have uniform distribution in liver and noticeably higher number of GNR specific conjugates in mouse tumor.

We present SS data showing the accumulation of PEG-GNR and GNR-HER2 into liver Kupffer cells in comparable concentration, as this is not related to selective binding: these cells are specialized macrophages located in the liver and lining the walls of the sinusoids, and are responsible for removing non metabolized compounds from the organism. They are an important part of the reticuloendothelial system [26].

We used GNR conjugates for IV administration in same dose as we studied for enhancement of optoacoustic imaging pegylated GNR:

PBS

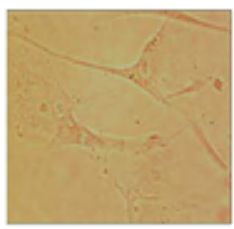

Fibroblasts + GNR Before Incubation with Blood

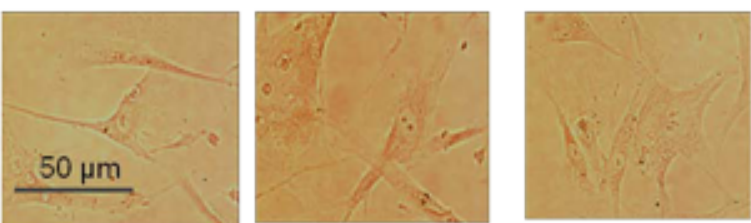

Fibroblast + GNR After Incubation with Blood
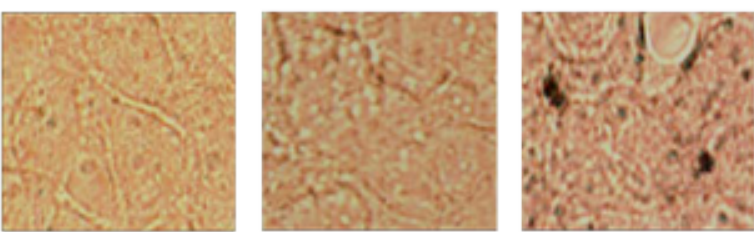

BT 474 + GNR Before Incubation with Blood
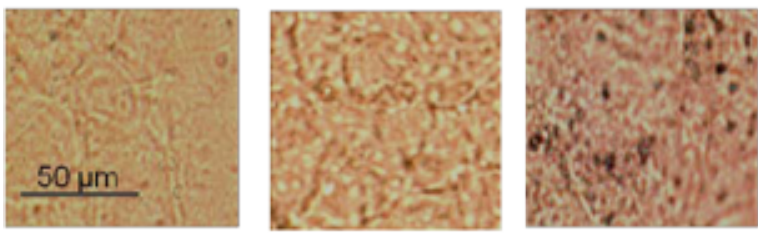

BT 474 + GNR After Incubation with Blood

Figure 9: Silver staining of fibroblast (no HER2/neu expression) and BT-474 cells following 60 min pre-treatment with pegylated (GNR-PEG) or conjugated through protocol 1 (GNR-PEG-HER2) GNR which were incubated with heparinized mouse blood for four hours. BT 474 shows silver enhancement for GNR-PEG-HER2 conjugates in both conditions: before and after incubation of GNR-conjugates with blood. 
PBS

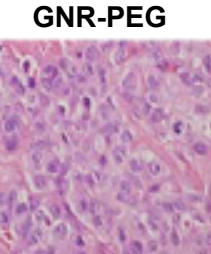

Tumor, H\&E
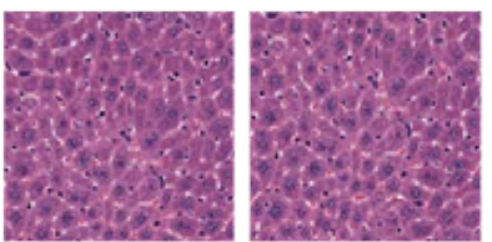

Liver, H\&E
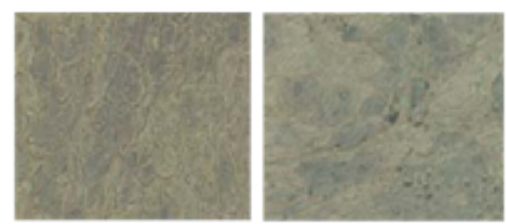

Tumor, SS
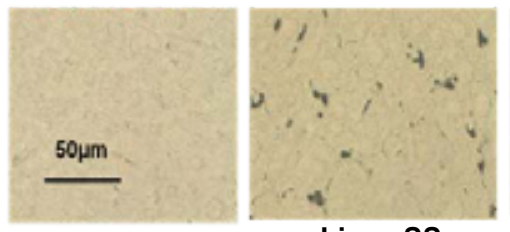

Liver, SS
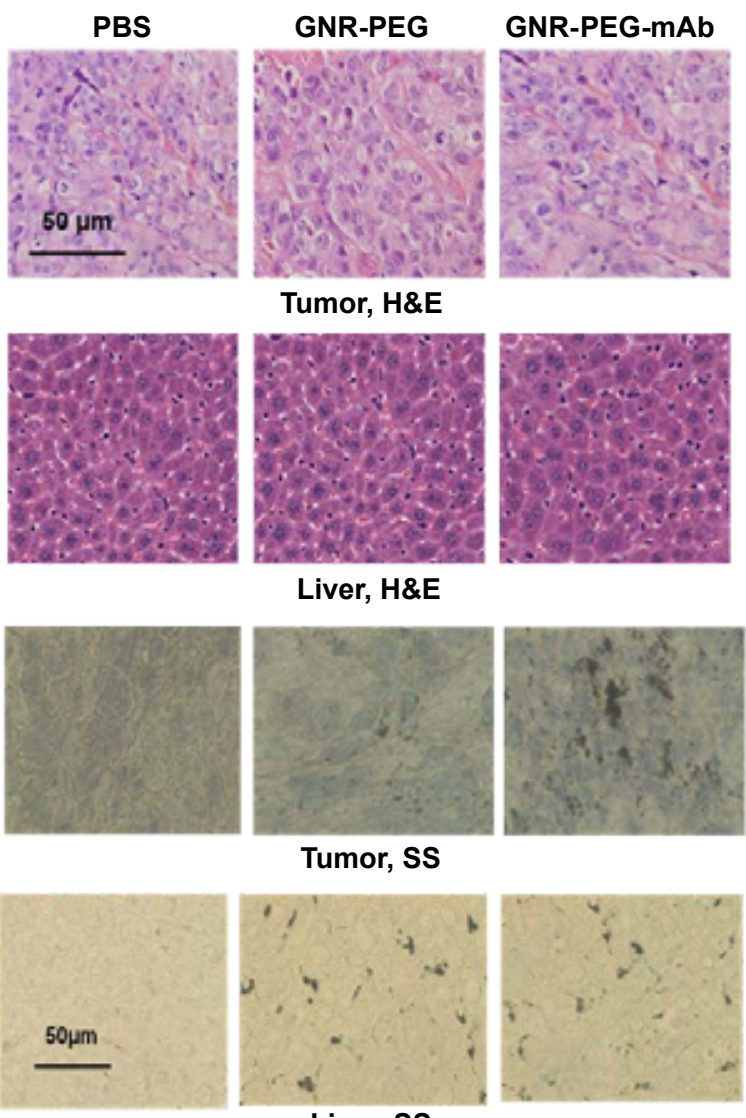

Figure 10: Above: Hematoxylin \& Eosin (tumor and liver), and Below: silver staining (tumor and liver) of GNR accumulated in mouse tissues following intravenous injection of PBS, GNR-PEG or GNR-PEG-HER2 conjugates Silver staining shows that GNR peg or Ab conjugates have uniform distribution in liver and noticeably higher number of GNR specific conjugates in mouse tumor.

around $20 \mathrm{mg} / \mathrm{kg} / \mathrm{BW}[15,16]$ we believe that specific binding can significantly improve medical imaging.

\section{Discussion and Summary}

Currently, conjugation of biomolecules to gold nanorods can be divided into four different methodologies: the use of a biofunctional linker which binds to gold at one end and to biomolecules on the other end, direct ligand exchange of the CTAB capping molecules with the biomolecules, functionalized coating of molecules that are electrostatically adsorbed onto the capping molecules while binding to biomolecules at their sites of functional groups, and electrostatically adsorbed onto the capping molecules [18].

The thiol exchange is the most common way to replace the original capping molecules since the metal-sulfur bond is known to be the strongest bond among different functional groups: amines, carboxylic acids, alcohols, and phosphors [18].

Biomolecules such as PEG, [9,21] DNA, [14,46], lipids [47] and small molecular ligands for cellular biomarkers, [48] are first functionalized with an alkythiolated linker and then bound to GNR through $\mathrm{Au}-\mathrm{S}$ bonds after few hours of reaction [18].
For some biomolecules, such as antibodies and proteins, thiolation is complicated by the fact that molecules are too large to reach the gold surface due to the dense packing of the CTAB double layers. The use of small bifunctional molecules such as 3-mercaptopropionic acid (MPA), 11-mercaptoundecanoic acids (MUDA), and 16-Mercaptohexadecanoic acid (MHDA) $[34,38,49,50]$ and cystamine [51] are useful in these studies. These small bifunctional molecules are very similar to bifunctional PEG.

Biocompatible and nontoxic nanoparticles have been used for in vivo tumor targeting and detection based on pegylated gold nanoparticles and surface-enhanced Raman scattering [52]. This team used two different molecules of PEG, carboxy PEG thiol (3000 Da) for $\mathrm{mAb}$ bonding and PEG thiol (500 DA) for preventing recognition and degradation by proteolytic enzymes uptake by the RES. Our preliminary study demonstrated that MHDA with pegylation is preferred for prevention of aggregation of GNR conjugates in solutions of BSA or FBS. The method of bifunctional linkage is easier, faster, and more efficient, in comparison with other methods of removal of CTAB and along with pegylation can be successfully used for long-time storage (two-three month) or in vivo application. Additionally, this method made better masks against recognition of GNR conjugates for in vivo application, because the "bridge" MHDA-zero size linker (EDC-NHS)$\mathrm{mAb}$ is comparable in size (length) as PEG thiol 5000. Furthermore, the functional activity of $\mathrm{Ab}$ is not lost upon conjugating. Accumulation of GNR conjugates in regions of interest can form a base for contrast enhancements in many applications such as optical imaging, opto- and photo- acoustic imaging, optical coherent tomography, thermotherapy and thermolysis, drug delivery and monitoring, as well as sensing.

In summary, we demonstrated that a combination of pegylation before conjugating GNR with $\mathrm{mAb}$ provided nontoxic GNR-PEGHER2 conjugates with stable plasmon resonance suitable for in vitro and in vivo applications. We successfully optimized a protocol for fabrication of GNR conjugates to be suitable as in vivo contrast agent. We have shown the optimal molar ratio between short functionalized molecules used as bridges for linkers, and methoxy PEG thiol was around 1 to 4 . During the conjugation step, the optimum ratio between activated GNR and number of molecules of $\mathrm{mAb}$ is around 1000. These ratios can be used for many biomolecules for conjugation with GNR for biomedical applications.

\section{Acknowledgements}

This work was supported by research grants from the National Institutes of Health R43ES021629; R44CA110137; R44CA110137-05S1 and SC3GM087201 to AK.

\section{References}

1. Liao H, Hafner JH (2005) Gold Nanorod Bioconjugates. Chem Mater 17: 46364641.

2. Perez-Juste J, Pastoria-Santos I, Liz-Marzan LM, Mulvaney P (2005) Gold nanorods: Synthesis, characterization, and applications. Coord Chem Rev 249: 1870-1901.

3. Alkilany AM, Murphy CJ (2010) Toxicity and cellular uptake of gold nanoparticles: what we have learned so far? J Nanopart Res 12: 2313-2333.

4. Huang X, El-Sayed IH, El-Sayed MA (2010) Applications of Gold Nanorods for Cancer Imaging and Photothermal Therapy. Methods Mol Biol 624: 343-357.

5. Tiwari PM, Vig K, Dennis VA, Singh SR (2011) Functionalized Gold Nanoparticles and Their Biomedical Applications. Nanomaterials 1: 31-63.

6. Oraevsky A (2009) Gold and silver nanoparticles as contrast agents for optoacoustic imaging, in Photoacoustic imaging and spectroscopy. Taylor and Francis Group, New York. 
Citation: Liopo A, Conjusteau A, Tsyboulski D, Ermolinsky B, Kazansky A, et al. (2012) Biocompatible Gold Nanorod Conjugates for Preclinical Biomedical Research. J Nanomedic Nanotechnol 6:274. doi:10.4172/2157-7439.1000.274

7. Khlebstov NG Dykman LA (2010) Optical properties and biomedical applications of plasmonic nanoparticles. J Quant Spectrosc Radiat Transf 111 $1-35$.

8. Jain KK (2010) Advances in the field of nanooncology. BMC Med 8: 83.

9. Dickerson EB, Dreaden EC, Huang X, El-Sayed IH, Chu H, et al. (2008) Gold nanorod assisted near infrared plasmonic photothermal therapy (PPTT) of squamous cell carcinoma in mice. Cancer lett 269: 57-66.

10. Urbanska K, Romanowska-Dixon B, Matuszak Z, Oszajca J, Nowak-Sliwinska $P$, et al. (2002) Indocyanine green as a prospective sensitizer for photodynamic therapy of melanomas. Acta Biochim Pol 49: 387-391.

11. Huang X, Jain PK, El-Sayed IH, El-Sayed MA (2007) Gold nanoparticles: interesting optical properties and recent applications in cancer diagnostics and therapy. Nanomedicine(Lond) 2: 681-693.

12. Huang X, Jain PK, El-Sayed IH, El-Sayed MA (2006) Determination of the minimum temperature required for selective photothermal destruction of cance cells with the use of immunotargeted gold nanoparticles. Photochem photobio 82: $412-417$

13. Bonoiu AC, Mahajan SD, Ding H, Roy I, Yong KT, et al. (2009) Nanotechnology approach for drug addiction therapy: gene silencing using delivery of gold nanorod-siRNA nanoplex in dopaminergic neurons. Proc Natl Acad Sci U S A 106: $5546-5550$

14. Chen CC, Lin YP, Wang CW, Tzeng HC, Wu CH, et al. (2006) DNA-gold nanorod conjugates for remote control of localized gene expression by near infrared irradiation. J Am Chem Soc 128: 3709-3715.

15. Su R, Ermilov SA, Liopo AV, Oraevsky AA (2012) Three-dimensional optoacoustic imaging as a new noninvasive technique to study long-term biodistribution of optical contrast agents in small animal models. J Biomed Op 17: 101506.

16. Liopo A, Conjusteau A, Chumakova O, Ermilov S, Su R, et al. (2012) Highly purified biocompatible gold nanorods for contrasted optoacoustic imaging of small animal models. Nanoscience and Nanotechnology Letters 4: 1-6.

17. Conjusteau A, Liopo A, Tsyboulski D, Ermilov S, Oraevsky AA, et al. (2011) Optoacoustic sensor for nanoparticle-linked immunoabsorbent assay (NanoLISA). Proc SPIE 7899:789910.

18. Huang X, Neretina S, El-Sayed MA (2009) Gold nanorods: from Synthesis and Properties to Biological and Biomedical Applications. Adv Mater 21: 4880-4910.

19. Liao CK, Huang SW, Wei CW, Li PC (2007) Nanorod-based flow estimation using a high-frame-rate photoacoustic imaging system. J Biomed Opt 12 064006 .

20. Chamberland DL, Agarwal A, Kotov N, Brian Fowlkes J, Carson PL, et al. (2008) Photoacoustic tomography of joints aided by an Etanercept-conjugated gold nanoparticle contrast agent-an ex vivo preliminary rat study. Nanotechnology 19: 095101.

21. Niidome T, Yamagata M, Okamoto $Y$, Akiyama $Y$, Takahashi $H$, et al. (2006) PEG-modified gold nanorods with a stealth character for in vivo applications. $J$ Control Release 114: 343-347.

22. Rostro-Kohanloo BC, Bickford LR, Payne CM, Day SE, Anderson LJ, et al. (2009) The stabilization and targeting of surfactant-synthesized gold nanorods. Nanotechnology 20: 434005.

23. Niidome T, Ohga A, Akiyama Y, Watanabe K, Niidome Y, et al. (2010) Controlled release of PEG chain from gold nanorods: targeted delivery to tumor. Bioorg Med Chem 18: 4453-4458.

24. Rayavarapu RG, Petersen W, Hartsuiker L, Chin P, Janssen H, et al. (2010) In vitro toxicity studies of polymer-coated gold nanorods. Nanotechnology 21 : 145101.

25. Weisbecker CS, Merritt MV, Whitesides GM (1996) Molecular Self- Assembly of Aliphatic Thiols on Gold Colloids. Langmuir 12: 3763-3772.

26. Roberts MJ, Bentley MD, Harris JM (2002) Chemistry for peptide and protein PEGylation. Adv Drug Deliv Rev 54: 459-476.

27. Liao H, Nehl CL, Hafner JH (2006) Biomedical applications of plasmon resonant metal nanoparticles. Nanomedicine (Lond) 1: 201-208.

28. Huang HC, Rege K, Heys JJ (2010) Spatiotemporal temprature distribution and cancer cell death in response to extracellular hyperthermia induced by gold nanorods. ACS Nano 4: 2892-2900.
29. Chen S, Ji Y, Lian $Q$, Wen $Y$, Shen $H$, et al. (2010) Gold Nanorods Coated with Multilayer Polyelectrolyte as Intracellular delivery Vector of Antisense Oligonucleotides. Nano Biomed Eng 2: 15-23.

30. Zhang X, Pan B, Wang K, Ruan J, Bao C, et al. (2010) Electrochemical Property and Cell Toxicity of Gold Electrode Modified by Monolayer PAMAM Encapsulated Gold Nanorods. Nano Biomed Eng 2: 182-188.

31. Xu W, Luo T, Pang B, Li PC, Zhou C, et al. (2012) The radiosensitization of melanoma cells by gold nanorods irradiated with MV-Xray. Nano Biomed Eng 4: 6-11.

32. Maltzahn G von, Park JH, Agrawal A, Bandaru NK, Das SK, et al. (2009) Computationally Guided Photothermal Tumor Therapy Using Long-Circulating Gold Nanorod Antennas. Cancer Res 69: 3892-3900.

33. Rayavarapu RG, Petersen W, Ungureanu C, Post JN, van Leeuwen TG, et al. (2007) Synthesis and Bioconjugation of Gold Nanoparticles as Potential Molecular Probes for Light-Based Imaging Techniques. Int J Biomed Imaging 2007.

34. Eghtedari M, Liopo AV, Copland JA, Oraevsky AA, Motamedi M (2009) Engineering of Hetero-Functional Gold Nanorods for the in vivo Molecular Targeting of Breast Cancer Cells. Nano Lett 9: 287-291.

35. Green HN, Martyshkin DV, Rodenberg CM, Rosenthal EL, Mirov SB (2011) Gold nanorods bioconjugates for active tumor targeting and photothermal therapy. Journal of Nanotechnology 2011: 1-7

36. Nikoobakht B, El-Sayed MA (2003) Preparation and Growth Mechanism of Gold Nanorods (NRs) Using Seed-Mediated Growth Method. Chem Mater 15 1957-1962.

37. Sau TK, Murphy CJ (2004) Seeded High Yield Synthesis of Short Au Nanorods in Aqueous Solution. Langmuir 20: 6414-6420.

38. Liopo AV, Conjusteau A, Konopleva M, Andreeff M, Oraevsky AA (2012) Laser nanothermolysis of human leukemia cells using functionalized plasmonic nanoparticles. Nano Biomed Eng 4: 66-75.

39. Liopo A, Conjusteau A, Oraevsky A (2012) PEG-coated gold nanorod monoclonal antobody conjugates in preclinical research with optoacoustic tomography, photothermal therapy, and sensing. Proc SPIE.

40. Chumakova OV, Liopo AV, Andreev VG, Cicenaite I, Evers BM, et al. (2008) Composition of PLGA and PEI/DNA nanoparticles improves ultrasoundmediated gene delivery in solid tumors in vivo. Cancer lett 261: 215-225.

41. Liopo AV, Stewart MP, Hudson J, Tour JM, Pappas TC (2006) Biocompatibility of native and functionalized single-walled carbon nanotubes for neuronal interface J Nanosci Nanotechnol 6: 1365-1374.

42. Alkilany AM, Nagaria PK, Wyatt MD, Murphy CJ (2010) Cation exchange on the surface of gold nanorods with a polymerizable surfactant: polymerization, stability and toxicity evaluation. Langmuir 26: 9328-9333.

43. Chumakova OV, Liopo AV, Evers BM, Esenaliev RO (2006) Effect of 5-fluorouracil, Optison and ultrasound on MCF-7 cell viability. Ultrasound Med Biol 32: 751-758.

44. MacDonald HR (2000) CD1d-glycolipid tetramers: A new tool to monitor natura killer T cells in health and disease. J Exp Med 192: F15-20.

45. Motamedi S, Shilagard T, Edward K, Koong L, Qui S, et al. (2011) Gold nanorods for intravital vascular imaging of preneoplastic oral mucosa. Biomed Opt Express 2: 1194-1203.

46. Dujardin E, Hsin LB, Wang CRC, Mann S (2001) DNA-driven self-assembly of gold nanorods. Chem Commun 1264-1265.

47. Nakashima H, Furukawa K, Kashimura Y, Torimitsu K (2008) Self-Assembly of Gold Nanorods Induced by Intermolecular Interactions of Surface-Anchored Lipids. Langmuir 24: 5654-5658.

48. Huff TB, Hansen MN, Zhao Y, Cheng JX, Wei A (2007) Controlling the cellular uptake of gold nanorods. Langmuir 23: 1596-1599.

49. Yu C, Varghese L, Irudayaraj J (2007) Surface modification of cetyltrimethylammonium bromide-capped gold nanorods to make molecular probes. Langmuir 23: 9114-9119.

50. Dai Q, Coutts J, Zou J, Huo Q (2008) Surface modification of gold nanorods 
Citation: Liopo A, Conjusteau A, Tsyboulski D, Ermolinsky B, Kazansky A, et al. (2012) Biocompatible Gold Nanorod Conjugates for Preclinical Biomedical Research. J Nanomedic Nanotechnol 6:274. doi:10.4172/2157-7439.1000.274

Page 10 of 10

through a place exchange reaction inside an ionic exchange resin. Chem Commun (Camb) 2858-2860.

51. Li PC, Wang CR, Shieh DB, Wei CW, Liao CK, et al. (2008) In vivo photoacoustic molecular imaging with simultaneous multiple selective targeting using antibody-conjugated gold nanorods. Opt Express 16: 18605-18615.

52. Qian X, Zhou X, Nie S (2008) Surface-enhanced Raman nanoparticle beacons based on bioconjugated gold nanocrystals and long range plasmonic coupling J Am Chem Soc 130: 14934-14935. 